\title{
Respiratory System Findings Reference Result in Original Units
}

National Cancer Institute

\section{Source}

National Cancer Institute. Respiratory System Findings Reference Result in Original Units. NCI Thesaurus. Code C162214.

The reference result for continuous respiratory measurements in original units. 